\title{
Multifunction between bitopologcal spaces via ideals
}

\author{
B. Brundha ${ }^{1 *}$ and P. Gomathi sundari ${ }^{2}$
}

\author{
Abstract \\ In this paper, we introduce and study some properties of pairwise upper (lower) semi- $\mathscr{I}$-continuous multifunctions. \\ Keywords \\ Ideal bitopological spaces, $(i, j)$-semi- $\mathscr{I}$-open sets, $(i, j)$-semi- $\mathscr{I}$-continuous multifunctions. \\ AMS Subject Classification \\ 54A40. \\ ${ }^{1}$ Department of Mathematics, Government Arts College for Women, Affiliated to Bharathidasan University, Orathanadu-614625, Tamil Nadu, \\ India. \\ ${ }^{2}$ Department of Mathematics, Rajah Serfoji Government College, Affiliated to Bharathidasan University, Thanjavur-613006, Tamil Nadu, India. \\ *Corresponding author: ${ }^{*}$ brindamithunraj@gmail.com; ${ }^{2}$ rsgcgomathi18@gmail.com \\ Article History: Received 16 October 2019; Accepted 07 December 2019 \\ (C)2020 MJM
}

\section{Contents}

Introduction $\ldots \ldots \ldots \ldots \ldots \ldots \ldots \ldots \ldots \ldots \ldots \ldots \ldots \ldots, 54$

2 Preliminaries $\ldots \ldots \ldots \ldots \ldots \ldots \ldots \ldots \ldots \ldots \ldots \ldots \ldots$

3 On $(i, j)$-semi- $\mathscr{I}$-continuous multifunctions .......55

References ............................... 57

\section{Introduction}

It is well known that various types of functions play a significant role in the theory of classical point set topology. A great number of papers dealing with such functions have appeared, and a good number of them have been extended to the setting of multifunctions [8-11]. This implies that both, functions and multifunctions are important tools for studying other properties of spaces and for constructing new spaces from previously existing ones. The concept of ideals in topological spaces has been introduced and studied by Kuratowski [6] and Vaidyanathaswamy, [13]. An ideal $\mathscr{I}$ on a topological space $(X, \tau)$ is a nonempty collection of subsets of $X$ which satisfies (i) $A \in \mathscr{I}$ and $B \subset A$ implies $B \in \mathscr{I}$ and (ii) $A \in \mathscr{I}$ and $B \in \mathscr{I}$ implies $A \cup B \in \mathscr{I}$. Given a bitopological space $\left(X, \tau_{1}, \tau_{2}\right)$ with an ideal $\mathscr{I}$ on $X$ and if $\mathscr{P}(X)$ is the set of all subsets of $X$, a set operator $(.)_{i}^{\star}: \mathscr{P}(X) \rightarrow \mathscr{P}(X)$, called the local function [13] of $A$ with respect to $\tau_{i}$ and $\mathscr{I}$, is defined as follows: for $A \subset X, A_{i}^{\star}\left(\tau_{i}, \mathscr{I}\right)=\{x \in X: U \cap A \notin \mathscr{I}$ for every $\left.U \in \tau_{i}(x)\right\}$, where $\tau_{i}(x)=\left\{U \in \tau_{i}: x \in U\right\}$. A Kuratowski closure operator $i \mathrm{Cl}^{\star}(\cdot)$ is defined by $i \mathrm{Cl}^{\star}(A)=A \cup A_{i}^{\star}\left(\tau_{i}, \mathscr{I}\right)$ when there is no chance of confusion, $A_{i}^{\star}(\mathscr{I})$ is denoted by
$A_{i}^{\star}$. If $\mathscr{I}$ is an ideal on $X$, then $\left(X, \tau_{1}, \tau_{2}, \mathscr{I}\right)$ is called an ideal bitopological space. In this paper, we introduce and study pairwise upper (lower) semi- $\mathscr{I}$-continuous multifunctions and obtain several characterizations of such functions.

\section{Preliminaries}

By a multifunction $F: X \rightarrow Y$, following [1], we shall denote the upper and lower inverse of a set $B$ of $Y$ by $F^{+}(B)$ and $F^{-}(B)$, respectively, that is, $F^{+}(B)=\{x \in X: F(x) \subset B\}$ and $F^{-}(B)=\{x \in X: F(x) \cap B \neq \emptyset\}$. A subset $S$ of an ideal bitopological space $\left(X, \tau_{1}, \tau_{2}, \mathscr{I}\right)$ is $(i, j)$-semi- $\mathscr{I}$-open [5] if $S \subset j \mathrm{Cl}^{\star}(i \operatorname{Int}(S))$. The complement of an $(i, j)$-semi- $\mathscr{I}$ closed set is said to be an $(i, j)$-semi- $\mathscr{I}$-open set. The $(i, j)$ semi- $\mathscr{I}$-closure and the $(i, j)$-semi- $\mathscr{I}$-interior, that can be defined in the same way as $\mathrm{Cl}(A)$ and $\operatorname{Int}(A)$, respectively, will be denoted by $(i, j)-s \mathscr{I} \mathrm{Cl}(A)$ and $(i, j)$-s $\mathscr{I} \operatorname{Int}(A)$, respectively. The family of all $(i, j)$-semi- $\mathscr{I}$-open (resp. $(i, j)$-semi$\mathscr{I}$-closed) sets of $\left(X, \tau_{1}, \tau_{2}, \mathscr{I}\right)$ is denoted by $(i, j)-S \mathscr{I} O(X)$ (resp. $(i, j)-S \mathscr{I} C(X))$. The family of all $(i, j)$-semi- $\mathscr{I}$-open (resp. $(i, j)$-semi- $\mathscr{I}$-closed) sets of $\left(X, \tau_{1}, \tau_{2}, \mathscr{I}\right)$ containing a point $x \in X$ is denoted by $(i, j)-S \mathscr{I} O(X, x)$ (resp. $(i, j)$ $S \mathscr{I} C(X, x))$. A subset A of a bitopolgical space $\left(X, \tau_{1}, \tau_{2}\right)$ is said to be $\tau_{1} \tau_{2}$-closed [2] if $A=\tau_{1}-\mathrm{Cl}\left(\tau_{2}-\mathrm{Cl}(A)\right)$. The complement of a $\tau_{1} \tau_{2}$-closed set is said to be $\tau_{1} \tau_{2}$-open. The intersection of all $\tau_{1} \tau_{2}$-closed sets containing $A$ is called $\tau_{1} \tau_{2}$ closure of $A$ and denoted by $\tau_{1} \tau_{2}-\mathrm{Cl}(A)$. The union of all $\tau_{1} \tau_{2}$-open sets contained in $A$ is called $\tau_{1} \tau_{2}$-interior of $A$ and denoted by $\tau_{1} \tau_{2}-\operatorname{Int}(A)$. 


\section{On $(i, j)$-semi- $\mathscr{I}$-continuous multifunctions}

Definition 3.1. A function $F:\left(X, \tau_{1}, \tau_{2}, \mathscr{I}\right) \rightarrow\left(Y, \sigma_{1}, \sigma_{2}\right)$ is said to be

1. upper $(i, j)$-semi- $\mathscr{I}$-continuous if for each $x \in X$ and each $\sigma_{1} \sigma_{2}$-open set $V$ of $Y$ containing $F(x)$, there exists $U \in(i, j)-S \mathscr{I} O(X, x)$ such that $F(U) \subset V$.

2. lower $(i, j)$-semi- $\mathscr{I}$-continuous if for each $\sigma_{1} \sigma_{2}$-open set $V$ of $Y$ containing $F(x)$ such that $F(x) \cap V \neq \emptyset$, there exists $U \in(i, j)-S \mathscr{I} O(X, x)$ such that $F(u) \cap V \neq \emptyset$ for every $u \in U$.

3. upper (lower) $(i, j)$-semi- $\mathscr{I}$-continuous if it has the property at each point of $X$.

Theorem 3.2. Let $F:\left(X, \tau_{1}, \tau_{2}, \mathscr{I}\right) \rightarrow\left(Y, \sigma_{1}, \sigma_{2}\right)$ be a multifuntion, the following statements are equivalent

1. F is upper $(i, j)$-semi- $\mathscr{I}$-continuous.

2. $F^{+}(V) \in(i, j)-S \mathscr{I} O(X)$ for any $\sigma_{1} \sigma_{2}$-open set $V$ of $Y$.

3. $F^{-}(V)$ is $(i, j)$-semi- $\mathscr{I}$-closed in $X$ for any $\sigma_{1} \sigma_{2}$-closed set $V$ of $Y$.

4. $F((i, j)-s \mathscr{I} \mathrm{Cl}(A)) \subset \sigma_{1} \sigma_{2}-\mathrm{Cl}(F(A))$ for each subset $A$ in $X$.

5. $(i, j)-s \mathscr{I} \mathrm{Cl}\left(F^{+}(B)\right) \subset F^{+}\left(\sigma_{1} \sigma_{2}-\mathrm{Cl}(B)\right)$ for each subset $B$ in $Y$.

Proof. The proof is clear.

Theorem 3.3. The following are equivalent for a multifunction $F:\left(X, \tau_{1}, \tau_{2}, \mathscr{I}\right) \rightarrow\left(Y, \sigma_{1}, \sigma_{2}\right)$

1. F is lower $(i, j)$-semi- $\mathscr{I}$-continuous.

2. $F^{-}(V) \in(i, j)-S \mathscr{I} O(X)$ for any $\sigma_{1} \sigma_{2}$-open set $V$ of $Y$.

3. $F^{+}(V)$ is $(i, j)$-semi- $\mathscr{I}$-closed in $X$ for any $\sigma_{1} \sigma_{2}$-closed set $V$ of $Y$.

4. $(i, j)-s \mathscr{I} \mathrm{Cl}\left(F^{+}(B)\right) \subset F^{+}\left(\sigma_{1} \sigma_{2}-\mathrm{Cl}(B)\right)$ for any $B \subset$ $Y$.

5. $F((i, j)-s \mathscr{I} \mathrm{Cl}(A)) \subset \sigma_{1} \sigma_{2}-\mathrm{Cl}(F(A))$ for any $A \subset X$.

Proof. Straightforward.

For a multifunction $F:(X, \tau) \rightarrow(Y, \sigma)$, the graph multifunction $G_{F}: X \rightarrow X \times Y$ is defined as follows $G_{F}(x)=$ $\{x\} \times F(x)$ for every $x \in X$.

Lemma 3.4. For a multifunction $F:(X, \tau) \rightarrow(Y, \sigma)$, the following hold:

$$
\text { 1. } G_{F}^{+}(A \times B)=A \cap F^{+}(B)
$$

2. $G_{F}^{-}(A \times B)=A \cap F^{-}(B)$ for any subsets $A \subset X$ and $B \subset Y$.

Definition 3.5. [2] A bitopological space $\left(X, \tau_{1}, \tau_{2}\right)$ is said to be $\tau_{1} \tau_{2}$-compact if every cover of $X$ by $\tau_{1} \tau_{2}$-open sets of $X$ has a finite subcover.

Theorem 3.6. Let $F:\left(X, \tau_{1}, \tau_{2}, \mathscr{I}\right) \rightarrow\left(Y, \sigma_{1}, \sigma_{2}\right)$ be a multifunction such that $F(x)$ is $\sigma_{1} \sigma_{2}$-compact for each $x \in X$. Then $F$ is upper $(i, j)$-semi- $\mathscr{I}$-continuous if and only if $G_{F}: X \rightarrow$ $X \times Y$ is upper $(i, j)$-semi- $\mathscr{I}$-continuous.

Proof. Let $x \in X$ and $W$ be any $\sigma_{1} \sigma_{2}$-open set of $X \times Y$ containing $G_{F}(x)$. For each $y \in F(x)$ there exist $\sigma_{1} \sigma_{2}$-open sets $U(y) \subset X$ and $V(y) \subset Y$ such that $(x, y) \in U(y) \times V(x) \subset W$. The family of $\{V(y): y \in F(x)\}$ is a $\sigma_{1} \sigma_{2}$-open cover of $F(x)$ and $F(x)$ is $\sigma_{1} \sigma_{2}$-compact. Then there exist a finite number of points, say $y_{1}, y_{2} \ldots, y_{n}$ in $F(x)$ such that $F(x) \subset$ $\cup\left\{V\left(y_{i}\right): 1 \leq i \leq n\right\}$. Set $U=\cap\left\{U\left(y_{i}\right): 1 \leq i \leq n\right\}$ and $V=\cup\left\{V\left(y_{i}\right): 1 \leq i \leq n\right\}$. Then $U$ and $V$ are $\sigma_{1} \sigma_{2}$-open in $X$ and $Y$ respectively and $\{x\} \times F(x) \subset U \times V \subset W$. Since $F$ is upper $(i, j)$-semi- $\mathscr{I}$-continuous, there exists $U_{0} \in(i, j)$ $S \mathscr{I} O(X, x)$ such that $F\left(U_{0}\right) \subset V$. By Lemma 3.4, we have $U \cap U_{0} \subset U \cap F^{+}(V)=G_{F}^{+}(U \times V) \subset G_{F}^{+}(W)$. Therefore, we obtain $U \cap U_{0} \in(i, j)-S \mathscr{I} O(X, x)$ and $G_{F}\left(U \cap U_{0}\right) \subset W$. This shows that $G_{F}$ is upper $(i, j)$-semi- $\mathscr{I}$-continuous. Conversely, suppose that $G_{F}: X \rightarrow X \times Y$ is upper $(i, j)$-semi$\mathscr{I}$-continuous. Let $x \in X$ and $V$ be any $\sigma_{1} \sigma_{2}$-open set of $Y$ containing $F(x)$. Since $X \times V$ is $\sigma_{1} \sigma_{2}$-open in $X \times Y$ and $G_{F}(x) \subset X \times V$, there exists $U \in(i, j)-S \mathscr{I} O(X, x)$ such that $G_{F}(U) \subset X \times V$. By Lemma 3.4 we have $U \subset G_{F}^{+}(X \times V)=$ $F^{+}(V)$ and $F(U) \subset V$. This shows that $F$ is upper $(i, j)$-semi$\mathscr{I}$-continuous.

Theorem 3.7. A multifunction $F:\left(X, \tau_{1}, \tau_{2}, \mathscr{I}\right) \rightarrow\left(Y, \sigma_{1}, \sigma_{2}\right)$ is lower $(i, j)$-semi- $\mathscr{I}$-continuous if and only if $G_{F}: X \rightarrow$ $X \times Y$ is lower $(i, j)$-semi- $\mathscr{I}$-continuous.

Proof. Suppose that $F$ is lower $(i, j)$-semi- $\mathscr{I}$-continuous. Let $x \in X$ and $W$ be any $\sigma_{1} \sigma_{2}$-open set of $X \times Y$ such that $x \in$ $G_{F}^{-}(W)$. Since $W \cap(\{x\} \times F(x)) \neq \emptyset$, there exists $y \in F(x)$ such that $(x, y) \in W$ and hence $(x, y) \in U \times V \subset W$ for some $\sigma_{1} \sigma_{2}$-open sets $U \subset X$ and $V \subset Y$. Since $F(x) \cap V \neq \emptyset$, there exits $G \in(i, j)-S \mathscr{I} O(X, x)$ such that $G \subset F^{-}(V)$. By Lemma 3.4, we have $U \cap G \subset U \cap F^{-}(V)=G_{F}^{-}(U \times V) \subset G_{F}^{-}(W)$. Moreover, $x \in U \cap G \in(i, j)-S \mathscr{I} O(X)$ and hence $G_{F}$ is a lower $(i, j)$-semi- $\mathscr{I}$-continuous. Conversely, suppose that $G_{F}$ is a lower $(i, j)$-semi- $\mathscr{I}$-continuous. Let $x \in X$ and $V$ be a $\sigma_{1} \sigma_{2}$-open set of $Y$ such that $x \in F^{-}(V)$. Then $X \times V$ is open in $X \times Y$ and $G_{F}(x) \cap(X \times V)=(\{x\} \times F(x)) \cap(X \times$ $V)=\{x\} \times(F(x) \cap V) \neq \emptyset$. Since $G_{F}$ is a lower $(i, j)$-semi$\mathscr{I}$-continuous, there exists $U \in(i, j)-S \mathscr{I} O(X, x)$ such that $U \subset G_{F}^{-}(X \times V)$. By Lemma 3.4, we obtain $U \subset F^{-}(V)$. This shows that $F$ is lower $(i, j)$-semi- $\mathscr{I}$-continuous.

Theorem 3.8. If $F:\left(X, \tau_{1}, \tau_{2}, \mathscr{I}\right) \rightarrow\left(Y, \sigma_{1}, \sigma_{2}\right)$ is an upper $(i, j)$-semi- $\mathscr{I}$-continuous multifunction such that $F(x)$ is $\sigma_{1} \sigma_{2}$-compact for each $x \in X$ and $Y$ is a $\sigma_{1} \sigma_{2}$-Housdorff 
space, then the multigraph $G(F)$ of $F$ is $(i, j)$-semi- $\mathscr{I}$-closed in $X \times Y$.

Proof. Let $(x, y) \notin G(F)$, that is, $y \notin F(x)$. Since $Y$ is $\sigma_{1} \sigma_{2}$ Housdorff, for each $z \in F(x)$, there exist disjoint $\sigma_{1} \sigma_{2}$-open sets $V(z)$ and $U(z)$ of $Y$ such that $z \in U(z)$ and $y \in V(z)$. Then $\{U(z): z \in F(x)\}$ is a $\sigma_{1} \sigma_{2}$-open cover of $F(x)$ and since $F(x)$ is $\sigma_{1} \sigma_{2}$-compact, there exits a finite number of points say, $s, z_{1}, z_{2}, \ldots z_{n}$ in $F(x)$ such that $F(x) \subset \cup\left\{U\left(z_{i}\right): i=\right.$ $1,2, \ldots n\}$. Put $U=\cup\left\{U\left(z_{i}\right): i=1,2, \ldots n\right\}$ and $V=\cap\left\{V\left(y_{i}\right)\right.$ : $i=1,2, \ldots n\}$. Then $U$ and $V$ are $\sigma_{1} \sigma_{2}$-open in $Y$ such that $F(x) \subset U, y \in V$ and $U \cap V=\emptyset$. Since $F$ is upper $(i, j)$-semi$\mathscr{I}$-continuous, there exist $W \in(i, j)-S \mathscr{I} O(X, x)$ such that $F(W) \subset U$. Since $V$ is $\sigma_{1} \sigma_{2}$-open, $W \times V \in(i, j)-S \mathscr{I} O(X \times$ $Y)$ and $(x, y) \in W \times V \subset X \times Y \backslash G(F)$. Then $X \times Y \backslash G(F)=$ $(x, y) \in X \times Y \backslash G(F)$ semi- $\mathscr{I}$-closed in $X \times Y$.

For any two multifunctions $F_{1}: X_{1} \rightarrow Y_{1}$ and $F_{2}: X_{2} \rightarrow Y_{2}$, the following hold:

1. $\left(F_{1} \times F_{2}\right)^{+}(A \times B)=F_{1}^{+}(A) \times F_{2}^{+}(B)$

2. $\left(F_{1} \times F_{2}\right)^{-}(A \times B)=F_{1}^{-}(A) \times F_{2}^{-}(B)$ for any $A \subset X_{1}$ and $B \subset X_{2}$.

Lemma 3.9. If $F_{1}: X_{1} \rightarrow Y_{1}$ and $F_{2}: X_{2} \rightarrow Y_{2}$ are upper $(i, j)$ semi- $\mathscr{I}$-continuous (resp. lower $(i, j)$-semi- $\mathscr{I}$-continuous) multifunctions, then $F_{1} \times F_{2}: X_{1} \times X_{2} \rightarrow Y_{1} \times Y_{2}$ is upper $(i, j)$ semi- $\mathscr{I}$-continuous (resp. lower $(i, j)$-semi- $\mathscr{I}$-continuous).

Proof. We prove only the case for $F$ upper $(i, j)$-semi- $\mathscr{I}$ continuous. Let $\left(x_{1}, x_{2}\right) \in X_{1} \times X_{2}$ and $W$ be any $\sigma_{1} \sigma_{2}$-open set of $Y_{1} \times Y_{2}$ containing $F_{1}\left(x_{1}\right) \times F_{2}\left(x_{2}\right)$. There exist $\sigma_{1} \sigma_{2}-$ open sets $U$ and $V$ of $Y_{1}$ and $Y_{2}$ respectively, such that $F_{1}\left(x_{1}\right) \times$ $F_{2}\left(x_{2}\right) \subset U \times V \subset W$. Since $F_{1}$ and $F_{2}$ are upper $(i, j)$-semi$\mathscr{I}$-continuous, there exist $U_{0} \in(i, j)-S \mathscr{I} O\left(X_{1} . x_{1}\right)$ and $V_{0} \in$ $(i, j)-S \mathscr{I} O\left(X_{2}, x_{2}\right)$ such that $F_{1}\left(U_{0}\right) \subset U$ and $F_{2}\left(V_{0}\right) \subset V$. Then we have $U_{0} \times V_{0} \subset F_{1}^{+}(U) \times F_{2}^{+}(V)=\left(F_{1} \times F_{2}\right)^{+}(U \times$ $V) \subset\left(F_{1} \times F_{2}\right)^{+}(W)$. Therefore, we obtain $U_{0} \times V_{0} \in(i, j)$ $S \mathscr{I} O\left(X_{1} \times X_{2},\left(x_{1}, x_{2}\right)\right)$ and $\left(F_{1} \times F_{2}\right)^{+}\left(U_{0} \times V_{0}\right) \subset W$. This shows that $F_{1} \times F_{2}$ is upper $(i, j)$-semi- $\mathscr{I}$-continuous.

Definition 3.10. [2] A collection $\mathscr{U}$ of subsets of a bitopological space $\left(X, \tau_{1}, \tau_{2}\right)$ is said to be $\tau_{1} \tau_{2}$-locally finite if every $x \in X$ has a $\tau_{1} \tau_{2}$-neighborhood which intersects only finitely many elements of $\mathscr{U}$.

Definition 3.11. [2] A subset A of a bitopological space $\left(X, \sigma_{1}, \sigma_{2}\right)$ is said to be

1. $\sigma_{1} \sigma_{2}$-paracompact if every cover of $A$ by $\sigma_{1} \sigma_{2}$-open sets of $X$ is refined by a cover of $A$ which consists of $\sigma_{1} \sigma_{2}$-open set of $X$ and is $\sigma_{1} \sigma_{2}$-locally finite in $X$.

2. $\sigma_{1} \sigma_{2}$-regular if for each $a \in A$ and each $\sigma_{1} \sigma_{2}$-open set $U$ of $X$ containing a, there exists a $\sigma_{1} \sigma_{2}$-open set $G$ of $X$ such that $a \in G \subset \sigma_{1} \sigma_{2}-\mathrm{Cl}(G) \subset U$.
Lemma 3.12. [2] If $A$ is a $\tau_{1} \tau_{2}$-regular $\tau_{1} \tau_{2}$-paracompacat set of a bitopological space $\left(X, \tau_{1}, \tau_{2}\right)$ and $U$ is a $\tau_{1} \tau_{2}$-open neighbourhood of $A$, then there exists a $\tau_{1} \tau_{2}$-open set $G$ of $X$ such that $A \subset G \subset \tau_{1} \tau_{2}-\mathrm{Cl}(G) \subset U$.

Remark 3.13. [2] For a multifunction $F:\left(X, \tau_{1}, \tau_{2}, \mathscr{I}\right) \rightarrow$ $\left(Y, \sigma_{1}, \sigma_{2}\right)$, by $\mathrm{Cl} F:\left(X, \tau_{1}, \tau_{2}\right) \rightarrow\left(Y, \sigma_{1}, \sigma_{2}\right)$ we denote a multifunction defined as follows $\mathrm{Cl} F(x)=\tau_{1} \tau_{2}-\mathrm{Cl}(F(x))$ for each $x \in X$.

Lemma 3.14. [2] If $F:\left(X, \tau_{1}, \tau_{2}, \mathscr{I}\right) \rightarrow\left(Y, \sigma_{1}, \sigma_{2}\right)$ is a multifunction such that $F(x)$ is $\tau_{1} \tau_{2}$-paracompact $\tau_{1} \tau_{2}$-regular for each $x \in X$, then for each $\sigma_{1} \sigma_{2}$-open set $V$ of $Y, G^{+}(V)=$ $F^{+}(V)$, where $G$ denotes $\mathrm{Cl} F$.

Theorem 3.15. Let $F:\left(X, \tau_{1}, \tau_{2}, \mathscr{I}\right) \rightarrow\left(Y, \sigma_{1}, \sigma_{2}\right)$ be a multifunction such that $F(x)$ is a $\sigma_{1} \sigma_{2}$-paracompact and $\sigma_{1} \sigma_{2}$ regular for each $x \in X$. Then the following statements are equivalent.

1. $F$ is upper $(i, j)$-semi- $\mathscr{I}$-continuous.

2. $(i, j)$-s $\mathscr{I} \mathrm{Cl} F$ is upper $(i, j)$-semi- $\mathscr{I}$-continuous.

3. $\mathrm{Cl} F$ is upper $(i, j)$-semi- $\mathscr{I}$-continuous.

Proof. We put $G=(i, j)-s \mathscr{I} \mathrm{Cl} F$ or $\mathrm{Cl} F$ in the sequel. Suppose that $F$ is upper $(i, j)$-semi- $\mathscr{I}$-continuous. Let $x \in X$ and $V$ be any $\sigma_{1} \sigma_{2}$-open set of $Y$ containing $G(x)$. By Lemma 3.14, we have $x \in G^{+}(V)=F^{+}(V)$ and hence there exists $U \in$ $(i, j)-S \mathscr{I} O(X, x)$ such that $F(U) \subset V$. Since $F(u)$ is $\sigma_{1} \sigma_{2}-$ paracompact and $\sigma_{1} \sigma_{2}$-regular for each $u \in U$ by Lemma 3.12, there exists a $\sigma_{1} \sigma_{2}$-open set $W$ such that $F(u) \subset W \subset \sigma_{1} \sigma_{2}$ $\mathrm{Cl}(W) \subset V$; hence $G(u) \subset \sigma_{1} \sigma_{2}-\mathrm{Cl}(W) \subset V$ for each $u \in U$. Therefore, we obtain $G(U) \subset V$. This shows that $G$ is upper $(i, j)$-semi- $\mathscr{I}$-continuous. Conversely, suppose that $G$ is upper $(i, j)$-semi- $\mathscr{I}$-continuous. Let $x \in X$ and $V$ be any $\sigma_{1} \sigma_{2}$-open set of $Y$ containing $F(x)$ By Lemma 3.14, we have $x \in F^{+}(V)=G^{+}(V)$ and hence $G(x) \subset V$ there exist $U \in(i, j)-S \mathscr{I} O(X, x)$ such that $F(U) \subset V$. Therefore, we obtain $U \subset G^{+}(V)=F^{+}(V)$ and hence $F(U) \subset V$. This shows that $F$ is upper $(i, j)$-semi- $\mathscr{I}$-continuous.

Lemma 3.16. [2] If $F:\left(X, \tau_{1}, \tau_{2}, \mathscr{I}\right) \rightarrow\left(Y, \sigma_{1}, \sigma_{2}\right)$ is a multifunction then for each $\sigma_{1} \sigma_{2}$-open set $V$ of $Y . G^{-}(V)=F^{-}(V)$ where $G$ denotes $(i, j)$-s $\mathscr{I} \mathrm{Cl} F$ or $\mathrm{Cl} F$.

Theorem 3.17. The following are equivalent for a multifunction $F:\left(X, \tau_{1}, \tau_{2}, \mathscr{I}\right) \rightarrow\left(Y, \sigma_{1}, \sigma_{2}\right)$ :

1. $F$ is lower $(i, j)$-semi- $\mathscr{I}$-continuous.

2. $(i, j)$-s $\mathscr{I} \mathrm{Cl} F$ is lower $(i, j)$-semi- $\mathscr{I}$-continuous.

3. $\mathrm{Cl} F$ is lower $(i, j)$-semi- $\mathscr{I}$-continuous.

Proof. By using Lemma 3.16 this is shown similarly to that of Theorem 3.15. 
Definition 3.18. The $(i, j)$-semi- $\mathscr{I}$-frontier of a subset $A$ of an ideal bitopological space $\left(X, \tau_{1}, \tau_{2}, \mathscr{I}\right)$, denote by $(i, j)$ $s \mathscr{I} \operatorname{Fr}(A)$, is defined by $(i, j)-s \mathscr{I} F r(A)=(i, j)-s \mathscr{I} \mathrm{Cl}(A) \cap$ $(i, j)-s \mathscr{I} \mathrm{Cl}(X \backslash A)=(i, j)-s \mathscr{I} \mathrm{Cl}(A) \backslash(i, j)-s \mathscr{I} \operatorname{Int}(A)$.

Theorem 3.19. The set of all points of $X$ at which a multifunction $F:\left(X, \tau_{1}, \tau_{2}, \mathscr{I}\right) \rightarrow\left(Y, \sigma_{1}, \sigma_{2}\right)$ is not upper (lower) $(i, j)$-semi-I $\mathscr{I}$-continuous is identical with the union of the semi- $\mathscr{I}$-frontier of the upper (lower) inverse images of open sets containing (meeting ) $F(x)$.

Proof. Suppose that $x \in X$ at which $F$ is not upper $(i, j)$ semi- $\mathscr{I}$-continuous. Then there exists a $\sigma_{1} \sigma_{2}$-open set $V$ of $Y$ containing $F(x)$ such that $U \cap\left(X \backslash F^{+}(x)\right) \neq \emptyset$ for every $U \in(i, j)-S \mathscr{I} O(X, x)$. Then $x \in(i, j)-s \mathscr{I} \mathrm{Cl}\left(X \backslash F^{+}(V)\right)=$ $X \backslash(i, j)-s \mathscr{I} \operatorname{Int}\left(F^{+}(V)\right)$ and $x \in F^{+}(V)$. Hence we obtain $x \in$ $(i, j)$-s $\mathscr{I} F r\left(F^{+}(V)\right)$. Conversely, suppose $V$ is $\sigma_{1} \sigma_{2}$-open set of $Y$ containing $F(x)$ such that $x \in(i, j)-s \mathscr{I} F r\left(F^{+}(V)\right)$. If $F$ is upper $(i, j)$-semi- $\mathscr{I}$-continuous at $x$, there exists $U \in(i, j)$ $S \mathscr{I} O(X, x)$ such that $U \subset F^{+}(V)$. It is clear that $x \in(i, j)$ $s \mathscr{I} \operatorname{Int}\left(F^{+}(V)\right)$. This is a contradiction and hence $F$ is not $(i, j)$-upper semi- $\mathscr{I}$-continuous at $x$.

In the following $(D,>)$ is directed set, $\left(F_{\lambda}\right)$ is a net of multifunction $F_{\lambda}:\left(X, \tau_{1}, \tau_{2}\right) \rightarrow\left(Y, \sigma_{1}, \sigma_{2}\right)$ for every $\lambda \in D$ and $F$ is a multifunction from $X$ into $Y$.

Definition 3.20. Let $\left(F_{\lambda}\right)_{\lambda \in D}$ be a net of multifunctions from $\left(X, \tau_{1}, \tau_{2}\right)$ to $\left(Y, \sigma_{1}, \sigma_{2}\right)$. A multifunction $F^{*}:\left(X, \tau_{1}, \tau_{2}\right) \rightarrow$ $\left(Y, \sigma_{1}, \sigma_{2}\right)$ is defined as for each $x \in X, F^{*}(x)=\{y \in Y:$ for each $\sigma_{1} \sigma_{2}$-open neighbourhood $V$ of $y$ and each $\mu \in D$, there exist $\lambda \in D$ such that $\lambda>\mu$ and $\left.V \cap F_{\lambda}(x) \neq \emptyset\right\}$ is called the $\sigma_{1} \sigma_{2}$-topological limit of the net $\left(F_{\lambda}\right)_{\lambda \in D}$.

Definition 3.21. A net $\left(F_{\lambda}\right)_{\lambda \in D}$ is said to be equally upper $(i, j)$-semi-I $\mathscr{I}$-continuous at $x_{0} \in X$ if for every $\sigma_{1} \sigma_{2}$-open set $V_{\lambda}$ containing $F_{\lambda}\left(x_{0}\right)$, there exists $U \in(i, j)-S \mathscr{I} O\left(X, x_{0}\right)$ such that $F_{\lambda}(U) \subset V_{\lambda}$ for all $\lambda \in D$.

Theorem 3.22. Let $\left(F_{\lambda}\right)_{\lambda \in D}$ be a net of multifunction from an ideal bitopological space $\left(X, \tau_{1}, \tau_{2}, \mathscr{I}\right)$ into a $\sigma_{1} \sigma_{2}$-compact space $\left(Y, \sigma_{1}, \sigma_{2}\right)$. If the following are satisfied.

1. $\cup\left\{F_{\mu}(x): \mu>\lambda\right\}$ is $\sigma_{1} \sigma_{2}$-closed in $Y$ for each $\lambda \in D$ and each $x \in X$.

2. $\left(F_{\lambda}\right)_{\lambda \in D}$ is equally upper $(i, j)$-semi- $\mathscr{I}$-continuous on $X$, then $F^{*}$ is upper $(i, j)$-semi- $\mathscr{I}$-continuous on $X$.

Proof. From definition and (1) we have $F^{*}(x)=\cap\left(\left\{\cup F_{\mu}(x)\right.\right.$ : $\mu>\lambda\}: \lambda \in D)$. Since the net $\left(\cup\left\{F_{\mu}(x): \mu>\lambda\right\}\right)_{\lambda \in D}$ is a family of $\sigma_{1} \sigma_{2}$-closed sets having the finite intersection property and $Y$ is $\sigma_{1} \sigma_{2}$-compact, $F^{*}(x) \neq \emptyset$ for each $x \in X$. Now, let $x_{0} \in X$ and let $V$ be a proper $\sigma_{1} \sigma_{2}$-open subset of $Y$ such that $F^{*}\left(x_{0}\right) \subset V$. Since $F^{*}\left(x_{0}\right) \cap(Y \backslash V)=\emptyset, F^{*}\left(x_{0}\right) \neq \emptyset$ and $(Y \backslash V) \neq \emptyset, \cap\left\{\left(\cup\left\{F_{\mu}\left(x_{0}\right): \mu>\lambda\right\}\right): \lambda \in D\right\} \cap(Y \backslash V)=\emptyset$ and hence $\cap\left\{\left(\cup\left\{F_{\mu}\left(x_{0}\right) \cap(Y \backslash V): \mu>\lambda\right\}\right): \lambda \in D\right\}=\emptyset$. Since $Y$ is $\sigma_{1} \sigma_{2}$-compact and the family $\left\{\left(\cup\left\{F_{\mu}\left(x_{0}\right) \cap(Y \backslash V): \mu>\right.\right.\right.$ $\lambda\}): \lambda \in D\}$ is a family of $\sigma_{1} \sigma_{2}$-closed sets with the empty intersection, there exit $\lambda \in D$ such that $F_{\mu}\left(x_{0}\right) \cap(Y \backslash V)=\emptyset$ for each $\mu \in D$ with $\mu>\lambda$. Since the net $\left(F_{\lambda}\right)_{\lambda \in D}$ is equally upper $(i, j)$-semi- $\mathscr{I}$-continuous on $X$, there exists $U \in(i, j)$ $S \mathscr{I} O\left(X, x_{0}\right)$ such that $F_{\mu}(U) \subset V$ for each $\mu>\lambda$ that is, $F_{\mu}(x) \cap(Y \backslash V)=\emptyset$ for each $x \in U$. Then $\cup\left\{F_{\mu}(x) \cap(Y \backslash V)\right.$ : $\mu>\lambda\}=\emptyset$ and hence $\cap\left\{\left(\cup\left\{F_{\mu}(x) \cap(Y \backslash V): \mu>\lambda\right\}\right): \lambda \in\right.$ $D\} \cap(Y \backslash V)=\emptyset$. This implies that $F^{*}(U) \subset V$. If $V=Y$, then it is clear that for each $U \in(i, j)-S \mathscr{I} O\left(X, x_{0}\right)$ we have $F^{*}(U) \subset V$. Hence $F^{*}$ is upper $(i, j)$-semi- $\mathscr{I}$-continuous at $x_{0}$. Since $x_{0}$ is arbitrary, the proof completes.

Theorem 3.23. If $F:\left(X, \tau_{1}, \tau_{2}, \mathscr{I}\right) \rightarrow\left(Y, \sigma_{1}, \sigma_{2}\right)$ is upper $(i, j)$-semi- $\mathscr{I}$-continuous, point $\sigma_{1} \sigma_{2}$-closed and $Y$ is $\sigma_{1} \sigma_{2}$ regular, then the graph $G(F)$ of $F$ is $(i, j)$-semi- $\mathscr{I}$-closed set in the product space $X \times Y$.

Proof. Suppose $(x, y) \notin G(F)$. Then we have $y \notin F(x)$. Since $Y$ is $\sigma_{1} \sigma_{2}$-regular, there exist disjoint $\sigma_{1} \sigma_{2}$-open sets $V_{1}$ and $V_{2}$ of $Y$ such that $y \in V_{1}$ and $F(x) \subset V_{2}$. Since $F$ is upper $(i, j)$ semi- $\mathscr{I}$-continuous, $F^{+}\left(V_{2}\right)$ is $(i, j)$-semi- $\mathscr{I}$-closed set in $X$ containing $x$. Therefore, we obtain $(x, y) \in U \times F^{+}\left(V_{2}\right) \subset$ $X \times Y \backslash G(F)$ and so $G(F)$ is $(i, j)$-semi- $\mathscr{I}$-closed.

Theorem 3.24. If $F:\left(X, \tau_{1}, \tau_{2}, \mathscr{I}\right) \rightarrow\left(Y, \sigma_{1}, \sigma_{2}\right)$ is an upper (lower) $(i, j)$-semi- $\mathscr{I}$-continuous, point $\sigma_{1} \sigma_{2}$-closed and $Y$ is $\sigma_{1} \sigma_{2}$-normal, then $A=\left\{\left(x_{1}, x_{2}\right): F\left(x_{1}\right)=F\left(x_{2}\right)\right\}$ is $(i, j)$ semi-I $\mathscr{I}$-closed in the product space $X \times Y$.

Proof. Let $\left(x_{1}, x_{2}\right) \notin A$, then $F\left(x_{1}\right) \neq F\left(x_{2}\right)$. Since $Y$ is $\sigma_{1} \sigma_{2}$ normal, there exist disjoint $\sigma_{1} \sigma_{2}$-open sets $V_{1}$ and $V_{2}$ of $Y$ such that $F\left(x_{1}\right) \subset V_{1}$ and $F\left(x_{2}\right) \subset V_{2}$. Since $F$ is upper $(i, j)$-semi$\mathscr{I}$-continuous, $F^{+}\left(V_{1}\right)$ and $F^{+}\left(V_{2}\right)$ are $(i, j)$-semi- $\mathscr{I}$-open sets and $x_{1} \in F^{+}\left(V_{1}\right)$ and $x_{2} \in F^{+}\left(V_{2}\right)$. Therefore $\left[F^{+}\left(V_{1}\right) \times\right.$ $\left.F^{+}\left(V_{2}\right)\right] \cap A=\emptyset$. Since $\left(x_{1}, x_{2}\right) \in\left[F^{+}\left(V_{1}\right) \times F^{+}\left(V_{2}\right)\right]$ and $F^{+}\left(V_{1}\right) \times F^{+}\left(V_{2}\right)$ is $(i, j)$-semi- $\mathscr{I}$-open set in $X \times Y$, we ob$\operatorname{tain}\left(x_{1}, x_{2}\right) \in(i, j)-s \mathscr{I} \mathrm{Cl} A$.

\section{References}

[1] T. Banzaru, Multifunctions and $M$-product spaces, Bull. Stin. Tech. Inst. Politech. Timisoara, Ser. Mat. Fiz. Mer. Teor. Apl., 17(31)(1972), 17-23.

[2] C. Boonpok, C. Viriyapong, M. Thongmoon, On upper and lower $\left(\tau_{1}, \tau_{2}\right)$-precontinuous multifunctions, J. Math. Computer Sci., 18 (2018), 282?293.

[3] D. Jankovic and T. R. Hamlett, Compatible extension of ideals, Bull. U. M. I., 7(1992), 453-465.

[4] D. Jankovic and T. R. Hamlett, New toplogies from old via ideals, Amer. Math. Monthly, 97 (4) (1990), 295-310.

[5] M. Caldas, S. Jafari and N. Rajesh, Semiopen sets in ideal bitopological spaces (submitted).

[6] K. Kuratowski, Topology, Academic Press, New York, 1966.

[7] R. L. Newcomb, Topologies which are compact modulo an ideal, Ph.D. Thesis, University of California, USA(1967). 
[8] T. Noiri and V. Popa, Almost weakly continuous multifunctions, Demonstratio Math.,26 (1993), 363-380.

[9] T. Noiri and V. Popa, A unified theory of almost continuity for multifunctions, Sci. Stud. Res. Ser. Math. Inform., 20(1) (2010),185-214.

[10] V. Popa, A note on weakly and almost continuous multifunctions, Univ, u Novom Sadu, Zb. Rad. Prirod-Mat. Fak. Ser. Mat., 21(1991),31-38.

[11] V. Popa, Weakly continuous multifunction, Boll. Un. Mat. Ital., (5) 15-A(1978),379-388.

[12] M. Stone, Applications of the theory of boolean rings to general topology, Trans. Amer. Math. Soc., 41(1937), 374-381.

[13] R. Vaidyanathaswamy, The localisation theory in set topology, Proc. Indian Acad. Sci., 20(1945), 51-61.

ISSN(P):2319-3786

Malaya Journal of Matematik

ISSN(O):2321 - 5666

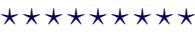

